\title{
Copy number variation and genetic diversity of MHC Class IIb alleles in an alien population of Xenopus laevis
}

\author{
Barbara K. Mable ${ }^{1}$ (D) $\cdot$ Elizabeth Kilbride $^{1} \cdot$ Mark E. Viney $^{2} \cdot$ Richard C. Tinsley $^{2}$
}

Received: 14 May 2015 / Accepted: 10 July 2015 /Published online: 2 September 2015

(C) The Author(s) 2015. This article is published with open access at Springerlink.com

\begin{abstract}
Xenopus laevis (the African clawed frog), which originated through hybridisation and whole genome duplication, has been used as a model for genetics and development for many years, but surprisingly little is known about immune gene variation in natural populations. The purpose of this study was to use an isolated population of $X$. laevis that was introduced to Wales, UK in the past 50 years to investigate how variation at the MHC compares to that at other loci, following a severe population bottleneck. Among 18 individuals, we found nine alleles based on exon 2 sequences of the Class IIb region (which includes the peptide binding region). Individuals carried from one to three of the loci identified from previous laboratory studies. Genetic variation was an order of magnitude higher at the MHC compared with three single-copy nuclear genes, but all loci showed high levels of heterozygosity and nucleotide diversity and there was not an excess of homozygosity or decrease in diversity over time that would suggest extensive inbreeding in the introduced population. Tajima's $D$ was positive for all loci, which is consistent with a bottleneck. Moreover, comparison with published sequences identified the
\end{abstract}

Electronic supplementary material The online version of this article (doi:10.1007/s00251-015-0860-3) contains supplementary material, which is available to authorized users.

Barbara K. Mable

Barbara.mable@glasgow.ac.uk

Mark E. Viney

Mark.Viney@bristol.ac.uk

1 Institute of Biodiversity, Animal Health \& Comparative Medicine, College of Medical, Veterinary \& Life Sciences, University of Glasgow, Glasgow G12 8QQ, UK

2 School of Biological Sciences, University of Bristol, Bristol BS8 1TQ, UK source of the introduced population as the Western Cape region of South Africa, where most commercial suppliers have obtained their stocks. These factors suggest that despite founding by potentially already inbred individuals, the alien population in Wales has maintained substantial genetic variation at both adaptively important and neutral genes.

Keywords Xenopus laevis $\cdot$ Introduced species $\cdot$ Polyploidy · MHC $\cdot$ Bottlenecks $\cdot$ Genetic diversity

\section{Introduction}

A major concern for conservation is how changing climates and human-mediated habitat alterations affect genetic diversity in increasingly fragmented and isolated populations. Although alien species are often viewed negatively, in cases where the introduced population is not able to invade a wide proportion of the new habitat, they can make useful models for investigating the genetic consequences of population fragmentation arising from introduction.

Xenopus laevis, the African clawed frog, is native to subSaharan Africa but has been introduced to multiple locations worldwide. This species was used for human pregnancy tests until the 1970s, as well as a model for developmental biology and genetics (Gurdon 1996). Alien X. laevis populations are established in many parts of the world, attributed to release of individuals originating from the southwest region of the Western Cape Province of South Africa (Lillo et al. 2013; Lillo et al. 2011; Measey et al. 2012; Tinsley and Kobel 1996), which has a Mediterranean climate. Populations have become established in various regions with a temperate climate, including the UK. In Wales, an alien, but closed, breeding population of $X$. laevis was founded about 50 years ago, prior to its extinction in 2010 (Tinsley et al. 2015b). Mark- 
recapture data were collected from this population for 30 years, with records of birth dates, gender, population size and infections with the monogenean Protopolystoma xenopodis (Tinsley et al. 2012). This population therefore provides an interesting model to examine how adaptation to a novel environment can impact genetic diversity of a fragmented population that was likely founded by a small number of individuals.

A complicating but intriguing aspect of $X$. laevis biology is that it is a tetraploid, meaning that it arose through genome duplication, possibly following a hybridisation event (allopolyploidisation). The whole genome duplication (WGD) event is thought to have occurred 29-66 mya (Chain and Evans 2006), and there has subsequently been substantial loss of duplicate gene expression, although many genes are retained in duplicate (Chain et al. 2011; Hellsten et al. 2007; Morin et al. 2006; Semon and Wolfe 2008). Differential loss or retention of duplicate genes could alter the consequences of genetic bottlenecks, or the ability to expand into new environments. In plants, polyploidy has been associated with invasiveness (defined as the ability to become established in new environments) due to the increased genomic flexibility that is predicted to accompany WGD (Ainouche et al. 2009; Pandit et al. 2011).

It has been proposed that $X$. laevis has played a role in the spread of important amphibian diseases such as the chytrid fungus (Weldon et al. 2004) and ranavirus (Robert et al. 2007), even though individuals do not normally present disease symptoms. One possibility for this is that duplication of genes coding for the immune machinery (or differential loss of duplicate copies) has conferred increased tolerance and/or resistance to pathogens and parasites (Jackson and Tinsley 2001; Jackson and Tinsley 2003). Even in diploids, duplication and divergence of genes coding for aspects of immune function is thought to have been important for generating redundant copies that can be recruited for tissue- or pathogenspecific defence, but also for enabling rapid expansion of the available repertoire through nonreciprocal recombination among copies (i.e. gene conversion; Martinsohn et al. 1999; Ohta 1991; Spurgin et al. 2011; van Oosterhout 2009). This has resulted in extensive copy number variation $(\mathrm{CNV})$ between species, within species and even within populations of diploid organisms (Llaurens et al. 2012). While tandem duplications certainly have contributed, the 'big bang' theory suggests that historic multiple rounds of WGD have resulted in the most dramatic changes in copy number of immune genes (reviewed by Flajnik and Kasahara 2010). Polyploids could thus be more resistant to parasites compared to their diploid progenitors because of greater allelic diversity of molecules of the immune system (Jackson and Tinsley 2001, 2003; King et al. 2012). In addition, the process of hybridisation could expand immune gene capacity through potentially advantageous interactions between genes encoded by each subgenome (Comai et al. 2000; Evans 2007). Alternatively, the development of new polyploids could substantially perturbate the immune repertoire, possibly leading to immunopathology, including due to autoimmunity (Zou and Secombes 2011). In plants, for instance, polyploids are sometimes, but not always, more resistant to attack by parasites than their diploid progenitors (Nuismer and Thompson 2001; Schoen et al. 1991; Thompson et al. 2004). An intriguing possibility is that this might depend on whether immune genes are retained in duplicate or dynamic loss of duplicate copies within or between populations, but this has not been investigated.

Despite being one of best studied ectothermic vertebrate models of immune function (e.g. Chida et al. 2011; Maniero et al. 2006; Robert and Cohen 2011; Robert and Ohta 2009), surprisingly little is known about natural variation in immune genes in $X$. laevis, or how immune gene variation is impacted by population bottlenecks in nature or inbreeding in laboratory colonies. This is reflected in lack of a standardised nomenclature for MHC genes in Xenopus. Some tetraploid (X. laevis) and octoploid (Xenopus vestitus) species express the same number of Major Histocompatibility Complex (MHC) copies as a closely related diploid species (Xenopus tropicalis), suggesting loss of copy number. In contrast, a more recently diverged dodecaploid species (Xenopus ruwenzoriensis) shows expression of additional copies, which suggests retention of duplicates (Du Pasquier and Blomberg 1982; Flajnik 1996; Kobari et al. 1995; Kobel and Du Pasquier 1986; Sammut et al. 2002; Sato et al. 1993; Shum et al. 1993). The MHC Class IIb region of $X$. laevis is organised into three loci, none of which appears to be retained in duplicate (Kobari et al. 1995). There are no published data available on variation at the MHC from multiple individuals sampled from a wild population of X. laevis, so it is not known whether MHC copy number is a fixed or variable trait. Published sequences also do not include information on heterozygosity of individuals because the focus has been on characterising the structure of the MHC based on partially inbred laboratory-reared animals (Klein et al. 2002; Kobari et al. 1995; Sato et al. 1993).

However, there has been comparison of variation both in mitochondrial (Evans et al. 1997) and single-copy nuclear genes (Bewick et al. 2011; Furman et al. 2015) for multiple individuals sampled from discrete populations in the native range of $X$. laevis in Africa, including the Western Cape Province of South Africa. Sequencing the same sets of genes in the alien Welsh population would allow an assessment of relative diversity at the MHC compared to other nuclear genes and to identify the likely source of the introduced population.

The overall purpose of this study was to use the Welsh $X$. laevis population as a model to investigate the impacts on genetic diversity of reproductive isolation in a novel alien habitat. We addressed four specific questions: (1) Is there evidence that the Class IIb region of the MHC is under diversifying selection? (2) Is there evidence for copy number variation at the MHC? (3) How much diversity and heterozygosity 
are maintained at the MHC compared to single-copy nuclear genes? (4) What was the likely source of the introduced population?

\section{Materials and methods}

\section{Samples, DNA extractions and PCR conditions}

The individuals used in this study were part of the long-term mark-recapture study described above (Additional file: Table S1; Tinsley et al. 2015a, b). Twelve of the 18 individuals sampled were born in 1993 (about 30 years after the initial population establishment), with the remainder born between 1999 and 2005; this sampling thus provides some perspective on changes in the population over time.

For the MHC, we targeted exon 2 of the Class IIb region using conserved primers described by Hauswaldt et al. (2007) that were designed for Rana temporaria but based on Xenopus spp. sequences (Haus-MHC-F: CCS CAG AKG ATT WCG TGW MTC A; Haus-MHC-5R: TGT CTG CAG ACT GTY TCC ACC HCA GCC). This exon contains most of the $\beta 1$ domain, which contains the peptide-binding region (PBR; Kobari et al. 1995). For comparison with published sequences from the native range in South Africa, we sequenced the mitochondrial 16S rDNA using the primers $16 \mathrm{Sc}$ (GTRGGCCTAAAAGCAGCCAC) and 16Sd (CTCCGGTCTGAACTCAGATCACGTAG) described in Moriarty and Cannatella (2004). We also sequenced three single-copy nuclear genes, using primers described in Bewick et al. (2011): two housekeeping genes (Prmt6, protein arginine methyltransferase; Xlaexon4_for1: GAC CRS GAG TAT TTC CAG TGC TAC TC; Xlaexon4_rev1: CAT AYG GCG ACG TMG ATA AAG TGA C and Mogs, mannosyloligosaccharide glucosidase; Xlaexon5_for2: CTG AAG ATG AGC GGC ATG TGG ATC TG; Xlaexon5_rev2: CTT CAG CCA TGA TTA GTA CCA C) and an immune - function - related coding gene not at the MHC (Rag2, recombination a c t ivating ge neX1a-R a g 2_for_4 5 : CTGGGAGTAATACATCATGATC; Xla-Rag2_rev_1149: CCTCGTCAAAATGTTCCCGTCTCTG). The three nuclear genes were chosen because: (1) they have been shown to be single copy in $X$. laevis and do not contain length variants, enabling resolution of heterozygous positions based on direct sequencing; (2) they were found to amplify reliably across all samples in initial tests; and (3) sequences were available in GenBank that included IUPAC ambiguity codes, enabling resolution of complete genotypes for heterozygous individuals and comparison with sequences from a broader geographic sampling (Furman et al. 2015).

Polymerase chain reactions were run using a standard set of conditions with Invitrogen Taq DNA polymerase (Invitrogen Inc., Paisley, UK) and its associated reagents, with annealing temperatures optimised for each set of primers $\left(58{ }^{\circ} \mathrm{C}\right.$ for MHC, $53{ }^{\circ} \mathrm{C}$ mtDNA, and $60{ }^{\circ} \mathrm{C}$ for other nuclear genes). Reactions were run in $20 \mu \mathrm{l}$ final volume using $2 \mu \mathrm{l}$ of $10 \times$ PCR buffer, $1 \mu \mathrm{l}$ of $50 \mathrm{mM} \mathrm{MgCl}, 2 \mu \mathrm{l} 2 \mathrm{mM}$ dNTPs (Promega Inc., Southampton, UK), $0.2 \mu \mathrm{l}$ of $500 \mathrm{U} / \mu \mathrm{l}$ Taq and $0.2 \mu \mathrm{l} 10 \mathrm{mM}$ stocks of each primer. Cycling conditions were $94{ }^{\circ} \mathrm{C}$ for $3 \mathrm{~min}$, annealing for $1 \mathrm{~min}, 72{ }^{\circ} \mathrm{C}$ for $2 \mathrm{~min}$, followed by 35 cycles of $94{ }^{\circ} \mathrm{C}$ for $30 \mathrm{~s}$, annealing for $30 \mathrm{~s}$, $72{ }^{\circ} \mathrm{C}$ for $2 \mathrm{~min}$, with a final extension at $72{ }^{\circ} \mathrm{C}$ for $6 \mathrm{~min}$.

For the MHC, amplicons were cloned into plasmid vectors using Invitrogen TOPO TA cloning kits for sequencing (Invitrogen Inc., Paisley, UK). A target of at least 18 clones per individual was set for sequencing of plasmids, purified using QIAprep Miniprep Kits (Qiagen Inc, Manchester, UK). Clones were bidirectionally sequenced by the GenePool, Edinburgh, using the Universal vector primers M13F and M13R. Chromatographs were assembled into contigs and base-calling errors corrected using Sequencher 4.0 (Gene Codes Corp, Ann Arbor, MI). Consensus sequences of sets of sequence variants within an individual were used to resolve alleles (referred to from this point on as haplotypes). In cases where a sequence variant was found in a single clone within an individual, this was only considered as a haplotype if also found in at least one other individual. The number of Class IIb haplotypes resolved per individual was plotted against the number of clones sequenced to determine whether it was likely that sequence variants might have missed (Additional file: Fig. S1).

For the other genes, amplification products were purified with USB Exosap-IT cleanup kits (Affymetrix Inc., USA) and sent to the GenePool in Edinburgh for direct sequencing in both directions. For the nuclear genes, haplotypes were resolved by eye, using the principle that the most common haplotype in a population was the basal sequence.

\section{Selection at the MHC}

BLAST was used to identify all other $X$. laevis sequences available in GenBank (Hauswaldt et al. 2007; Klein et al. 2002; Kobari et al. 1995; Sato et al. 1993), which were downloaded and aligned to the Welsh sequence variants (Fig. 1). All available sequences for MHC loci were from at least partially inbred laboratory-reared individuals (which we will refer to jointly as "laboratory colonies"). The translated amino acid sequence was compared to an amino acid alignment from Marsden et al. (2009) that compared variation in highly endangered African wild dogs to domestic dogs, as an indication of how sequence conservation in the MHC of the alien $X$. laevis population compared to other fragmented populations. Hypervariable regions (HVR) and antigen binding sites (ABS) were predicted based on characterisation of human HLA sequences (Bondinas et al. 2007). 


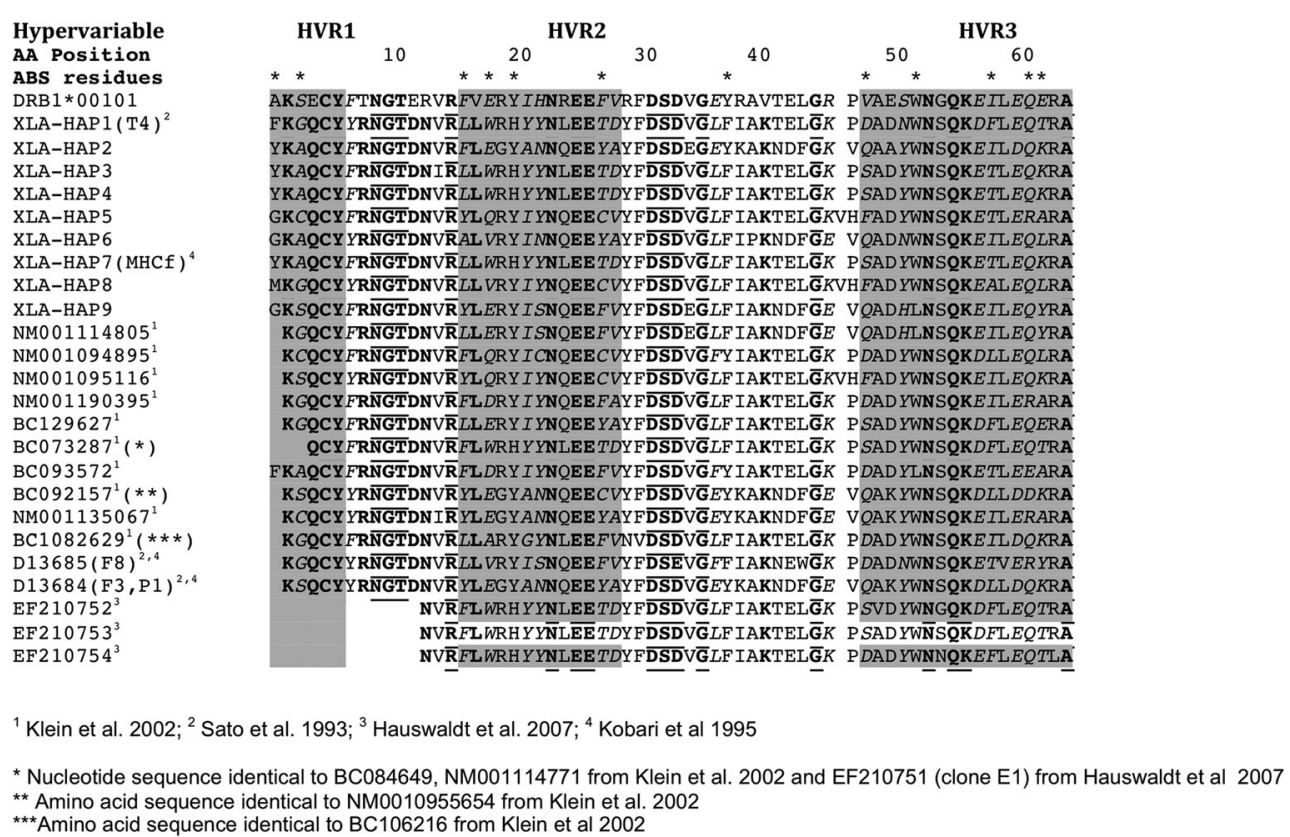

Fig. 1 Comparison of MHC Class IIb sequences from Welsh $X$. laevis (XLA-HAP), published laboratory $X$. laevis (accession number indicated) and a domestic dog sequence from the DRB1 locus (DRB1*00101). Hypervariable regions (HVR; shaded) and antigen binding sites (ABS, asterisks) are as defined in Marsden et al. (2009) for canid haplotypes based on human HLA sequences described in Bondinas et al. (2007). Amino acid numbers start from the beginning of the exon 2 region sequenced for Welsh Xenopus. Bold indicates amino acids conserved among all Xenopus haplotypes and underlined are those also conserved across highly divergent canid species (domestic dogs and African Wild

To test for selection acting on Class IIB alleles in relation to HVR and ABS predicted for humans, the datamonkey server (www.datamonkey.org), which implements statistical tests associated with the programme HyPhy (Pond et al. 2005), was used to analyse an alignment of all unique haplotypes in the Welsh populations with the laboratory sequences downloaded from GenBank. We used GARD (Genetic Algorithm for Recombination Detection; Pond et al. 2006) to test for evidence for recombination prior to using tests of selection. We chose the best fitting substitution model using CodonTest (Delport et al. 2010), and then various codon-based tests for selection were implemented. These are tree-based methods that compare the ratio of nonsynonymous changes per nonsynonymous site $\left(D_{n}\right)$ to synonymous changes per synonymous site $\left(D_{s}\right)$, such that ratios greater than one are indicative of positive selection and ratios less than one indicative of purifying selection. We compared sites predicted to be under positive selection by three codon-based tests for selection described in Pond and Frost (2005): (1) SLAC (Single Likelihood Ancestor Counting), FEL (Fixed Effects Likelihood) and REL (Random Effects Likelihood); (2) MEME (Mixed Effects Models of Evolution), which can detect evidence for diversifying and episodic selection (i.e. a large proportion of positively selected sites); and (3) IFEL (Internal Fixed Effects Likelihood), which investigates
Dogs; taken from Marsden et al. 2009). In total, 21/64 amino acids were conserved among dogs and all Xenopus, with one occurring in an HVR but none at predicted ABS. Residues found to be under positive selection using the REL method, as implemented in datamonkey, are indicated by italics; the strongest selection was found at sites 3 , 16 and 62, corresponding to one ABS in each of the HVR. Positive selection was detected at these sites for all other methods used as well (IFEL, FEL, MEME, SLAC). REL was the least conservative test, but sites 48 and 58 were also identified by most other methods; these are also at predicted ABS sites

population-level selection along internal branches. We also used BSR (Branch-Site REL; Pond et al. 2011) to investigate whether there was evidence for diversifying selection along particular branches of the genealogy.

Tests for deviations from neutrality based on allele frequencies of haplotypes within Wales were implemented using McDonald-Kreitman (McDonald and Kreitman 1991) and HKA tests (Hudson et al. 1987) in DNAsp version 5.10 (Librado and Rozas 2009), using sequences from $X$. tropicalis [as the outgroup because this was the most closely related species available in GenBank (NM_001045794)].

\section{MHC Class IIb copy number variation}

In order to separate sequences into predicted haplotypes according to the three MHC Class IIb loci that have been predicted previously from inbred laboratory-reared $X$. laevis $(D A B, D B B$ and $D C B$; Kobari et al. 1995), the nucleotide sequence alignment of the exon 2 region was used to reconstruct a genealogy using MEGA 6.0 (Tamura et al. 2013). ModelTest (as implemented in MEGA) was first used to select the best fitting model of evolution, and the maximum likelihood optimality criterion was used to reconstruct trees using the preferred substitution model, with confidence in branching relationships assessed using 1000 bootstrap pseudoreplications. 
No MHC Class IIb sequences were available in GenBank from other wild populations of Xenopus spp. Thus, haplotype summary statistics (as opposed to population estimates) computed using DNAsp version 5.10 (Librado and Rozas 2009) were used to compare the relative diversity in the three gene copies in Wales to that among published sequences from laboratory colonies, specifically, the number of haplotypes, number of segregating sites and average pairwise genetic diversity based on synonymous $\left(\pi_{\mathrm{s}}\right)$, nonsynonymous $\left(\pi_{\mathrm{n}}\right)$ and all sites $(\pi)$. Sequences were first collapsed into unique haplotypes for each sequence set and separate comparisons made within each for DAB, DBB, DCB and across all loci.

\section{MHC Class IIb variation compared to other genes}

For each gene, summary statistics based on complete genotypes for each individual were computed as for the MHC, but also included Watterson's $\theta$ (Watterson 1975). Tajima's $D$ statistic (Tajima 1989) was used to test whether there was evidence for deviation from neutral processes. This statistic compares diversity based on the total number of segregating sites (Watterson's $\theta$ ) and that based on the average number of mutations between pairs in the sample ( $\pi$; Nei 1987). Importantly, even in the absence of natural selection, Tajima's $D$ values are also affected by demographic changes (Fay and Wu 1999). An excess of low frequency polymorphisms (e.g. due to population size expansion after a bottleneck, a selective sweep or purifying selection) results in a negative value of $D$, whereas an excess of high frequency polymorphisms (e.g. due to a recent decrease in population size or balancing selection maintaining intermediate frequency variants) results in a positive value of $D$. McDonald-Kreitman and HKA tests were also conducted for each gene, as described for the MHC, using Xenopus gilli (accessions HQ221081, HQ221179, EF535964) as the outgroup because it is the most closely related species with sequences available in GenBank.

Since individuals had up to five haplotypes across all MHC Class IIb loci (Table 1), one minus the proportion of homozygous individuals was used as an estimate of heterozygosity that could be compared with the single-copy nuclear genes. In addition, observed $\left(H_{\mathrm{o}}\right)$ and expected $\left(H_{\mathrm{e}}\right)$ heterozygosity were calculated in Arlequin, version 3.5 (Excoffier and Lischer 2010), assuming complete diploid inheritance (i.e. only two copies of each allele per locus). These values were used to test whether observed heterozygosity deviated from that expected under Hardy-Weinberg Equilibrium (HWE) using Fisher's exact tests, as implemented in Arlequin.

Evidence for linkage disequilibrium between patterns of variation at the MHC and the other nuclear genes was investigated using contingency chi-squared analyses, with probability assessed through likelihood ratio tests (as implemented in JMP10.0; SAS Institute Inc. 2012) to assess whether: (1) individuals sharing genotypes at one of the other loci also shared $D A B$ genotypes; (2) there was an association between

Table 1 Haplotypes (indicated by assigned number) present for each of the three MHC Class IIb loci (DAB, DBB, DCB) in the Welsh population (complete individual names are provided in Additional file: Table S1, for comparison with Tinsley et al. 2012)

(


included representatives from many portions of sub-Saharan Africa (Evans et al. 2004) and so could be used to check the source of the released strains. For the single-copy nuclear genes, region of origin was also confirmed based on BLAST alignment to sequences available in GenBank and for two loci (Prmt6 and Mogs) to homologous sequences from X. laevis samples collected from over a dozen countries in sub-Saharan Africa (Furman et al. 2015).

\section{Results}

\section{Selection at the MHC}

A total of 14 MHC Class IIb haplotypes were identified among published sequences from laboratory colonies, two of which were shared with the Welsh population. The Welsh population had an additional seven haplotypes, which were similar, but not identical, to the published sequences. Among the Welsh samples, over the 64 codons (192 bp) examined, 40 were variable (comprising 69 variable nucleotide sites), which were distributed in nine haplotypes (GenBank accession numbers KP745469-77). Putative recombinant sequences were identified in five individuals, but these were assumed to be PCR recombinants as opposed to true haplotypes (Additional file: Table S2) and excluded from further analyses. Haplotypes 4 and 7 differed by four synonymous substitutions (all in third base pair positions), and haplotypes 3 and 4 differed from one another by one synonymous and one nonsynonymous substitution. The most divergent haplotypes (1 and 9) differed from one another by 38 nonsynonymous substitutions. Two individuals (11\%) were homozygous for haplotype 1; all other individuals had between two and five haplotypes. The number of haplotypes detected was not correlated with the number of clones sequenced (Additional file: Fig. S1 and Table S2).

Aligning these Welsh $X$. laevis sequences with those available in GenBank showed that there were 26 conserved and 38 variable amino acid residues in the sequenced region of the MHC, with 36 of the variable sites in predicted hypervariable regions (HVR) and 16 at predicted residues involved in peptide binding, only one of which is outside an HVR (Fig. 1). There was also a remarkable degree of conservation between $X$. laevis sequences and those from the canid samples described by Marsden et al. (2009). Evidence for positive selection was found at multiple codons using multiple tests for selection, despite the short length of the sequenced region, but these were concentrated at antigen binding sites (see Fig. 1). No strong evidence was found for strong diversifying selection overall, but there was some evidence for episodic selection along particular branches of the genealogy (Additional file: Fig. S4). Consistent with this, haplotype- based tests for selection (McDonald-Kreitman and HKA tests) were not significant.

\section{MHC Class IIb copy number variation}

The genealogy of the nine Welsh MHC-based haplotypes and all available sequences from GenBank (Fig. 2) was used to resolve the three Class IIb copies previously proposed (i.e. $D A B, D B B$ and $D C B$; Kobari et al. 1995), though not all nodes were strongly supported. $D A B$ sequences formed a monophyletic group with relatively shallow branch lengths (Additional file: Fig. S3) and included two Welsh haplotypes that were identical to published sequences from laboratory studies: haplotype 1 to clone T4 (Sato et al. (1993) and haplotype 7 to an MHCf homozygote (Kobari et al. (1995). Putative DBB sequences also formed a clearly resolved group but with higher among-haplotype divergence. None of these haplotypes was identical to published sequences, but haplotype 2 was most closely related to the $D B B$ 'type' of Kobari et al. (1995), and haplotype 6 was identical in amino acid sequence to NM00114805 from Klein et al. (2002). Putative $D C B$ sequences could not be classified so confidently, but this involved only two haplotypes (Fig. 2). All individuals had at least one of the four $D A B$ haplotypes (Additional file: Fig. S4), but presence of the other loci varied by individual (Table 1).

Summary statistics based on unique haplotypes (Table 2) showed that there was a substantial amount of genetic diversity at the MHC Class IIb, both within and between putative loci. Across all three loci, there was a similar level of synonymous diversity for Welsh and laboratory colony samples, but there tended to be lower nonsynonymous diversity among the Welsh samples. Across both sequence sets, there was consistently higher genetic distance between, rather than within, loci, with the largest difference between $D A B$ and $D B B$ (Additional file: Table S3). There were no substantial differences between $\pi_{\mathrm{n}}$ and $\pi_{\mathrm{s}}$ for any of the gene copies (Table 2), reflecting the overall conservation of polymorphism across the sequence and restriction of nonsynonymous variation to PBS (Fig. 1).

\section{MHC Class IIb variation compared to other genes}

The three single-copy nuclear genes were polymorphic (though an order of magnitude less variable compared with the MHC) and showed substantial heterozygosity in the Welsh population (Tables 3 and 4) (GenBank accession numbers KP745478531). For the housekeeping genes, five polymorphic sites were resolved into five distinct haplotypes for Prmt6 and three polymorphic sites were resolved into three distinct haplotypes for Mogs. Although Rag2 is a gene whose products have known immunological functions, only three polymorphic sites were found among the four haplotypes resolved. Haplotype diversity and number of haplotypes were comparable to that for $D A B$ at Prmt6 and Rag2, but Mogs showed lower variation (Table 3). 
Fig. 2 Mid-point rooted maximum likelihood genealogy for Class IIb sequences from Wales and published sequences, indicating the three putative loci described by Kobari et al. (1995); 'type' refers to the reference sequence for each locus reported in that paper. Bootstrap support (500 replicates) indicated when greater than $60 \%$. The Welsh sequences clearly cluster into DAB-like and DBB-like sequence types, but the remaining sequences do not resolve into a clearly distinctive grouping. For ease of comparison, they were designated as DCB. DAB-like genotypes segregated as expected for a single locus, but DCB (designated as Welsh haplotypes 5 and 8) and DBB were predominantly present as homozygotes. At least one sequence designated as DAB was present in all individuals sampled whereas DBB and DCB appeared to show presence/absence variation (PAV) among individuals (see Table 1)

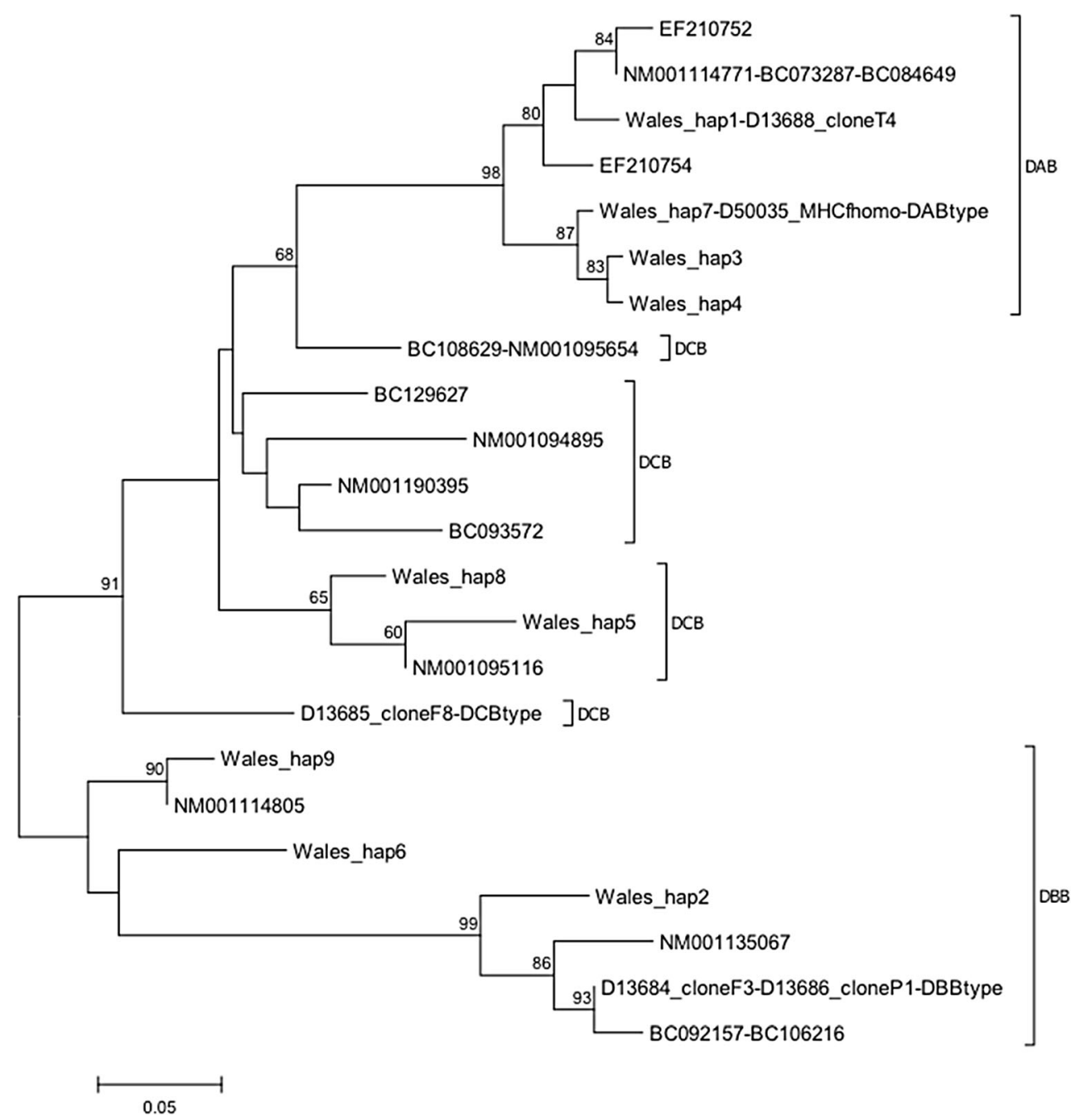

Gene diversity at synonymous, nonsynonymous and all sites showed an order of magnitude higher variation at DAB compared to the other nuclear genes. Heterozygosity at $D A B$ was lower than for Prmt6 and Rag2 but higher than Mogs. Tajima's $D$ was positive for all genes, reflected in the lower diversity based on Watterson's $\theta$ than pairwise nucleotide diversity $(\pi)$, and was significant for Mogs, Rag2 and DAB. When considered across all three MHC Class IIb loci, heterozygosity was higher than for any of the other nuclear genes and diversity was up to two orders of magnitude higher; Tajima's $D$ was positive but not significant, which might be expected if multiple loci were included in the same test. No significant deviation from neutrality was detected for any of the genes, using McDonaldKreitman or HKA tests.

Comparing observed $\left(H_{\mathrm{o}}\right)$ and expected $\left(H_{\mathrm{e}}\right)$ heterozygosity based on haplotype frequencies showed no evidence for excessive inbreeding in the Welsh samples (Additional file: Table S4). At $D A B, H_{\mathrm{o}}$ was less than $H_{\mathrm{e}}$, but the difference was not significant. Although the number of expected classes was small, DAB, Prmt6 and Rag2 segregated according to a model for a single diploid locus, with no deviations from
Hardy-Weinberg expectations based on the frequency of each haplotype. Observed heterozygosity was lower than expected at $\operatorname{Mogs}$, but the deviation was not significant.

There was little correspondence between the number of haplotypes (either at the MHC overall or $D A B$ separately) and heterozygosity across the other nuclear genes or between genotypes at the two sets of loci (Table 4). However, a $\chi^{2}$ square contingency test revealed a significant association between the number of $D A B$ haplotypes and heterozygosity at Rag2 (Additional file: Fig. S6c; $p=0.0016$ ): all $D A B$ heterozygotes were also heterozygous at Rag2 but some of the $D A B$ homozygotes were heterozygous at Rag2. There was no significant association for the other loci (Additional file: Fig. S6a, b).

There were no significant regressions between birth date (considered as a continuous trait) and the continuous response variables: (1) the number of Class IIb haplotypes, (2) the number of Class IIb loci, (3) the number of $D A B$ alleles, (4) withinindividual distances for Class IIb overall or $D A B$ separately and (5) within-individual distances for any of the single copy nuclear genes (Additional file: Table S5; Table 4). While all slopes were negative, they were extremely shallow and the 
Table 2 Comparison of genetic diversity based on unique haplotypes for new samples from Wales, published sequences from laboratory colonies (Lab) and considered across all (All), indicating number of unique haplotypes (Nhap); number of segregating sites (S); average pairwise genetic diversity based on synonymous $\left(\pi_{\mathrm{s}}\right)$, nonsynonymous $\left(\pi_{\mathrm{n}}\right)$ and all sites $(\pi)$

\begin{tabular}{lllllll}
\hline Locus & Pop & Nhap & $\mathrm{S}$ & $\pi_{\mathrm{s}}$ & $\pi_{\mathrm{n}}$ & $\pi$ \\
\hline DAB & Wales & 4 & 16 & 0.073 & 0.037 & 0.044 \\
& Lab & $5^{\mathrm{a}}$ & 13 & 0.012 & 0.048 & 0.040 \\
& All & 7 & 16 & 0.040 & 0.046 & 0.045 \\
DBB & Wales & 3 & 36 & 0.120 & 0.133 & 0.131 \\
& Lab & 4 & 32 & 0.062 & 0.104 & 0.095 \\
& All & 7 & 40 & 0.107 & 0.103 & 0.107 \\
DCB & Wales & 2 & 12 & 0.000 & 0.080 & 0.063 \\
& Lab & 7 & 43 & 0.057 & 0.104 & 0.094 \\
& All & 9 & 43 & 0.049 & 0.100 & 0.089 \\
All loci & Wales & 9 & 64 & 0.158 & 0.149 & 0.151 \\
& Lab & 16 & 65 & 0.154 & 0.166 & 0.162 \\
& All & 23 & 70 & 0.157 & 0.165 & 0.162 \\
\hline
\end{tabular}

${ }^{\mathrm{a}}$ Two published haplotypes were identical to those in Wales

model explained a very small amount of the variation. There also were no significant differences when birth date was considered by cohort (1993 vs. >1999) for any of these response variables (Additional file: Table S5), nor for the binomial response variables: (1) presence of DBB or DCB; and (2) heterozygosity of any of the genes (Additional file: Table S5).

\section{Source of the Welsh population}

Only one 890-bp mitochondrial 16S haplotype was recovered from the Welsh samples which, by BLAST analysis, was identical to a published sequence from an individual collected from the Western Cape Province of South Africa [accession AY581639, from X. laevis isolate Ig 12.3 (Evans et al. 2004); this sample was collected near Cape Town from Lewis Gay

Table 3 Comparison of summary statistics at the MHC with two housekeeping loci (Prmt6, Mogs) and an immune-related gene (Rag2), indicating number of individuals sequenced $(\mathrm{N})$; number of sequences compared (Ns); sequence length (L); number of segregating sites (S); number of haplotypes (Nhap); haplotype (gene) diversity; proportion of
Dam, Western Cape Province, South Africa]. This haplotype was also present in the areas of De Doorns and Hoekwil in the southwest Western Cape Province, South Africa (Furman et al. 2015). Except for a segment of a complete mitochondrial genome sequence (HM991335) from an individual collected from Jonkershoek, South Africa (which is $\sim 50 \mathrm{~km}$ from Cape Town), all other published sequences from wildcollected individuals from other parts of Africa were more substantially diverged and more distantly related (Additional file: Fig. S5). For the single-copy nuclear genes, the Welsh sequences also were identical to those found in the southwest Western Cape Province of SA (see Additional file: Fig. S2): (1) Garden Route National Park, De Doorns, and Laignsburg, for Prmt6; (2) Garden Route National Park, De Doorns, and Laignsburg, Beaufort West, Hoekwil, Niewoudtville and Betty's Bay for Mogs; and (3) Klapmuts, Blackheath and Betty's Bay for Rag2 (as well as sequences from individuals obtained from commercial suppliers).

\section{Discussion}

Our data provide the first assessment of MHC variation in a randomly breeding 'wild' population of Xenopus and suggest that copy number varies among individuals in this closed breeding population. Furthermore, we found that the introduced population of $X$. laevis in Wales has maintained substantial genetic diversity at both immune and housekeeping genes, despite potentially being founded by only a small number of individuals and isolated in a novel habitat for around 50 years.

\section{Selection at the MHC}

Despite the short length of the sequenced region (64 codons), multiple sites of the Class IIb region were found to be under positive selection, most of which were either in predicted HVR regions or at particular sites that have been implicated

individuals that were homozygous for each locus; average pairwise genetic diversity based on synonymous $\left(\pi_{\mathrm{s}}\right)$, nonsynonymous $\left(\pi_{\mathrm{n}}\right)$ and all sites $(\pi)$; and genetic diversity based on segregating sites $(\theta)$ and Tajima's $D$ statistic (significant values are indicated in bold)

\begin{tabular}{llllllllllll}
\hline Locus & $\mathrm{Ns}^{\mathrm{a}}$ & $\mathrm{L}$ & $\mathrm{S}$ & Nhap & Hap diversity & Pro. Homo & $\pi_{\mathrm{s}}$ & $\pi_{\mathrm{n}}$ & $\pi$ & $\theta$ & Tajima's $D$ \\
\hline Prmt6 & 36 & 642 & 5 & 5 & 0.78 & 0.17 & 0.0063 & 0.0021 & 0.0031 & 0.0019 & 1.65 \\
Mogs & 36 & 660 & 3 & 3 & 0.52 & 0.56 & 0.0063 & 0.0010 & 0.0022 & 0.0011 & $\mathbf{2 . 3 7}$ \\
Rag2 & 36 & 1007 & 3 & 4 & 0.68 & 0.28 & 0.0044 & 0.0005 & 0.0014 & 0.0007 & $\mathbf{2 . 0 9}$ \\
DAB & 39 & 192 & 16 & 4 & 0.73 & 0.39 & 0.0479 & 0.0333 & 0.0360 & 0.0200 & $\mathbf{2 . 5 7}$ \\
Class IIb & 53 & 192 & 64 & 9 & 0.87 & 0.11 & 0.1426 & 0.1200 & 0.1249 & 0.0991 & 0.91 \\
\hline
\end{tabular}

Summary statistics are shown for DAB alone and across all of the Class IIb haplotypes identified

${ }^{a}$ Note that the three single-copy nuclear genes behave as if diploid so there are two sequences per individual in the dataset; at the MHC, some individuals had more than two haplotypes and multiple copies, so there were 1-5 sequences per individual for these genes 
Table 4 Correspondence between genotypes across loci, showing the number of haplotypes per locus, with the MHC Class IIb indicated in terms of DAB alone, across all loci (Class IIb all) and which Class IIb loci were present; date of birth (DoB) of individuals is also indicated

\begin{tabular}{|c|c|c|c|c|c|c|c|}
\hline Individual & Prmt6 & Mogs & Rag2 & $D A B$ & Class IIb & Class IIb Loci & DoB \\
\hline 1 & 2 & 1 & 1 & 1 & 3 & $\begin{array}{c}\text { DAB, DBB, } \\
\text { DCB }\end{array}$ & 2003 \\
\hline 2 & 2 & 1 & 2 & 2 & 3 & $\mathrm{DAB}, \mathrm{DCB}$ & 1993 \\
\hline 3 & 2 & 1 & 2 & 2 & 2 & DAB & 2004 \\
\hline 4 & 2 & 2 & 1 & 1 & 1 & DAB & 1993 \\
\hline 5 & 2 & 1 & 1 & 1 & 3 & DCB & 1993 \\
\hline 6 & 2 & 1 & 2 & 2 & 3 & $\mathrm{DAB}, \mathrm{DCB}$ & 1993 \\
\hline 7 & 2 & 2 & 2 & 4 & 5 & DAB, DBB & 1993 \\
\hline 8 & 2 & 2 & 2 & 3 & 4 & DAB, DBB & 1993 \\
\hline 9 & 1 & 1 & 2 & 2 & 3 & DAB, DBB & 2003 \\
\hline & & & & & & DAB, DBB, & \\
\hline 10 & 2 & 1 & 2 & 2 & 4 & DCB & 1993 \\
\hline 11 & 2 & 1 & 2 & 3 & 3 & DAB & 1993 \\
\hline 12 & 2 & 2 & 2 & 1 & 1 & DAB & 2005 \\
\hline & & & & & & DAB, DBB, & \\
\hline 13 & 2 & 2 & 2 & 2 & 5 & DCB & 1999 \\
\hline 14 & 2 & 2 & 2 & 2 & 2 & DAB & 1993 \\
\hline 15 & 2 & 2 & 2 & 2 & 3 & DAB, DBB & 1993 \\
\hline 16 & 1 & 1 & 2 & 1 & 3 & $\begin{array}{l}\text { DAB, DBB, } \\
\text { DCB }\end{array}$ & 1993 \\
\hline 17 & 1 & 1 & 1 & 1 & 3 & DAB, DBB & 2002 \\
\hline 18 & 2 & 2 & 1 & 1 & 3 & $\begin{array}{l}\text { DAB, DBB, } \\
\text { DCB }\end{array}$ & 1993 \\
\hline
\end{tabular}

Colours indicate genotypes shared among individuals within loci (no colour indicates unique genotypes)

in peptide binding in humans (Bondinas et al. 2007). There was also evidence for episodic selection along branches, particularly those leading to Welsh haplotypes (Fig. 2). As expected for the MHC, there was substantial divergence among haplotypes when considered over all sequence variants (Fig. 1), despite the large conservation of amino acids that has been noted previously between Xenopus and humans (Ohta et al. 2006). For the population-level analyses within Wales, an excess of nonsynonymous diversity indicative of diversifying (positive) selection was restricted to particular codons involved in antigen recognition, with high conservation of amino acids in the rest of the gene, resulting in no significant selection in tests based on average values across the entire gene sequence (i.e. McDonald-Kreitman and HKA tests). This commonly observed pattern for genes coding for molecules with immune function (Chen et al. 2010; Garrigan and Hedrick 2001; Hughes et al. 1993; van Oosterhout et al. 2006) emphasises the importance of using codon-based tests for selection to assess evidence for positive selection, as most genes will include a combination of selected and conserved sites.

\section{MHC Class IIb copy number variation}

Interpreting the meaning of diversity at the MHC is complicated by separation of the Class IIb region into at least three loci (Kobari et al. 1995). Although the Welsh exon 2 sequences fell into three distinctive groups, the lack of resolution of the genealogy meant that sequences could only be assigned completely confidently to the $D A B$ locus. This locus was found in all individuals and segregated as a single diploid Mendelian locus. The presence of three haplotypes for some individuals at this locus contradicts findings based on laboratory colonies that both the Class I and Class II regions of the MHC are effectively diploid in tetraploid Xenopus, with physical loss of copies and disomic inheritance (Du Pasquier and Blomberg 1982; Kobari et al. 1995; Kobel and Du Pasquier 1986; Sato et al. 1993; Shum et al. 1993). Dodecaploid $X$. ruwenzoriensis have been shown to retain duplicate expression and show polysomic inheritance, suggesting that the locus is retained in duplicate at higher ploidy levels (Sammut et al. 2002). In contrast, for Lake Tana barbs (a group of fish that have undergone recent WGD), gene copy number increases with ploidy for class Ia but not class II genes (Kruiswijk et al. 2004). For the Welsh X. laevis samples, the third haplotype in each case (haplotype 7, which is identical to the $D A B$ type allele described by Kobari et al. 1995) is presumably functionally equivalent to haplotype 4 , from which it differed by only synonymous changes. Thus, this third haplotype could represent retention of a duplicate locus in some individuals or a recent tandem duplication. Haplotypes 3 and 4 differed from each other by only a single amino acid change that was outside of an HVR, but haplotype 1 was highly distinct from the other haplotypes (Additional file: Fig. S4). Therefore, functional diversity may not be as high as suggested by estimates based on nucleotide diversity.

The DBB and DCB loci were not found in all individuals, which suggests that they are 'expendable', despite including more highly divergent haplotypes than the $D A B$. We cannot completely rule out an amplification bias against these loci, 
but all three were amplified in one third of the individuals and this was not related to the number of clones sequenced; in fact, it tended to be individuals with fewer clones screened that showed amplification of all (Additional file: Table S2). It is also possible that there were divergent sequences at these loci that were not amplified. Nevertheless, presence/absence variation among genes coding for resistance phenotypes is common in plants (Bush et al. 2014; Shen et al. 2006) and copy number at the MHC among wild fish species and populations has been found to be extensive, even in diploids (Figueroa et al. 2001; Flajnik and Kasahara 2001; Milinski 2003; Murray et al. 2000; Sato et al. 1997). Eimes et al. (2011) also found that copy number varied within a highly bottlenecked population of prairie chickens, emphasising the dynamic nature of immune-related genes. Variation in copy number at the MHC has not previously been reported for Xenopus species, but most of the previous research on immunity has been based on inbred laboratory colonies, rather than natural populations (Klein et al. 2002; Kobari et al. 1995; Sato et al. 1993). It is possible that transfer of the Welsh population to the novel environment in the UK resulted in increased genetic drift, a relaxation of selection pressures to combat pathogens present in their habitats, or selection to maintain particular alleles in response to a novel disease agent. Alternatively, if maintenance of a high number of immune variants is costly, as suggested by the lack of retention of duplicate copies in polyploids and the finding of an optimal number of MHC alleles in fish (Forsberg et al. 2007; Milinski 2003; Reusch et al. 2001), relaxation of selection pressures could result in maintenance of a 'minimal set' of immune coding genes in the introduced alien population. Since we focused on a short fragment of exon 2, we cannot determine whether whole copies were lost or just this region, which would result in loss of function, as this is where most of the PBR resides (Kobari et al. 1995). Llaurens et al. (2012) suggested that focusing only on the exon containing the PBR could actually underestimate CNV and selection on the MHC since selection and gene conversion events could also occur outside of this region.

In the Welsh population, it is possible that copy number variation was present in the original founders. However, it is intriguing that homozygotes for a single haplotype (i.e. loss of $D B B$ and $D C B$ and homozygosity at $D A B$ ) are maintained in some individuals, whereas others retain all three loci. One of the homozygotes was born in the 1993 cohort and one in 2005, but neither showed records of parasites over the mark-recapture period (Additional file: Table S1). Similarly, four of the individuals that had all three Class IIb loci were born in 1993 and the other two were born in 1999 and 2003; four showed parasite presence and two did not, but both of the latter were born in 1993. It is tempting to predict an association with gender (both homozygotes were female, as were five out of six individuals with all three copies), but nearly twice as many females as males were sampled. The sample size is thus too small to draw definitive conclusions, but if copy number is a selectable trait, this could greatly affect the potential for species to adapt to novel environments or tolerate genetic bottlenecks.

\section{MHC Class IIb variation compared to other genes}

Within the Welsh $X$. laevis, there was no evidence for strong linkage disequilibrium across the three single-copy nuclear genes, and each showed slightly different patterns of variation. There was also no evidence for diversifying selection on these genes, in contrast to the MHC. However, there was an association between the number of $D A B$ haplotypes and heterozygosity at the other immune-related gene (Rag2). Gene diversity was an order of magnitude higher at $D A B$, but haplotype diversity was similar to that at Rag2. Although both genes showed significantly positive values of Tajima's $D$ that would be consistent with balancing selection, the observation of similar patterns at the housekeeping genes is more consistent with a signature of a recent demographic bottleneck (Fay and Wu 1999).

Combined with the overall high levels of heterozygosity observed at all loci and lack of deviation from HardyWeinberg expectations, the lack of an association between genetic variation at any of the genes compared with date of birth suggests that the population has not suffered from substantial inbreeding as a result of such a founder effect. This could suggest some mechanism for inbreeding avoidance. The MHC has been implicated in odour-based mate choice in a wide range of vertebrates (reviewed by Kamiya et al. 2014; Piertney and Oliver 2006), including humans (Potts et al. 1994; Roberts et al. 2005), other primates (Schwensow et al. 2008; Setchell et al. 2010), birds (Richardson et al. 2005; Zelano and Edwards 2002), fish (Blais et al. 2007; Eizaguirre et al. 2009; Forsberg et al. 2007; Neff et al. 2008; Reusch et al. 2001; Wedekind et al. 2004), salamanders (Bos et al. 2009) and reptiles (Miller et al. 2009). Higher levels of heterozygosity than expected based on neutral markers have also been observed in highly inbred island populations (e.g. killifish, Sato et al. 2002; Channel Island foxes, Aguilar et al. 2004). However, we did not find that the Class IIb region was maintained at a higher level of heterozygosity than the other loci screened, arguing that MHC-driven disassortative mating to avoid inbreeding has not occurred. Since many male anurans provide specific mating calls that females can use to discriminate between potential mates (Gerhardt 1991; Klump and Gerhardt 1987), there is the potential that females could use mating calls to distinguish genetically different mates, but this has not been tested in relation to genome-wide heterozygosity.

\section{Source of the Welsh population}

The identity of haplotypes at both mitochondrial and singlecopy nuclear genes with sequences sampled from the southwest Western Cape region of South Africa is consistent with 
previous suggestions that most introduced populations originated from animals originally collected from this region for scientific use or the pet trade (Lillo et al. 2013; Measey et al. 2012). This view is supported by haplotypes at the MHC class IIb that were identical to those from laboratory colonies, suggesting that founding could have been by a small number of released individuals, which could have already been partly inbred. It is thus even more intriguing that substantial heterozygosity and diversity has been preserved in a closed population for multiple generations. All of the genes used in this study have been predicted to be single copy in X. laevis, but segregation studies have not been conducted to determine whether they segregate consistently with complete diploid inheritance. It is possible that flexibility provided by multisomic inheritance could allow maintenance of higher levels of heterozygosity than would be expected for diploid organisms, even if only two copies are retained for each locus. Additional studies of patterns of inheritance would be required to specifically test whether the hybrid polyploid origin of $X$. laevis enables them to maintain higher levels of diversity than true diploids, despite extensive rediploidisation across the genome.

\section{Conclusions}

We have found a substantial amount of genetic diversity and heterozygosity maintained at both immune-related and housekeeping genes in the alien population of $X$. laevis from Wales. This is despite isolation in a novel environment and evidence for a genetic bottleneck. Importantly, our results are consistent with the results from other studies that copy number of MHC genes may be a variable trait within natural populations, which could have important implications for immune repertoire and flexibility in response to pathogens.

Acknowledgements We thank Robert Gillespie and Ryan Carter for assistance with laboratory work, and we thank the many field assistants acknowledged in Tinsley et al. (2012), especially Jenny York and Lucy Stott. Animals were individually marked in the mid-1990s by John Measey and in 1998-2002 by Joseph Jackson, whom we also thank. We particularly thank Ben Evans for access to unpublished data and for detailed comments on earlier versions of the manuscript. This work was funded by a grant to MEV from the UK Natural Environment Research Council (NE/H011498/1), including access to the NERC Biomolecular Analysis Facility at the GenePool in Edinburgh. Fieldwork was supported by grants from NERC and the UK Biotechnology and Biological Sciences Research Council (most recently BB/D523051/1).

Open Access This article is distributed under the terms of the Creative Commons Attribution 4.0 International License (http:// creativecommons.org/licenses/by/4.0/), which permits unrestricted use, distribution, and reproduction in any medium, provided you give appropriate credit to the original author(s) and the source, provide a link to the Creative Commons license, and indicate if changes were made.

\section{References}

Aguilar A, Roemer G, Debenham S, Binns M, Garcelon D, Wayne RK (2004) High MHC diversity maintained by balancing selection in an otherwise genetically monomorphic mammal. Proc Natl Acad Sci U S A 101:3490-3494

Ainouche ML et al (2009) Hybridization, polyploidy and invasion: lessons from Spartina (Poaceae). Biol Invasions 11:1159-1173

Bewick AJ, Anderson DW, Evans BJ (2011) Evolution of the closely related, sex-related genes Dm-W and Dmrt1 in African Clawed Frogs (Xenopus). Evolution 65:698-712

Blais J, Rico C, van Oosterhout C, Cable J, Turner GF, Bernatchez L (2007) MHC adaptive divergence between closely related and sympatric African cichlids. PLoS One 2, e734

Bondinas GP, Moustakas AK, Papadopoulos GK (2007) The spectrum of HLA-DQ and HLA-DR alleles, 2006: a listing correlating sequence and structure with function. Immunogenetics 59:539-553

Bos DH, Williams RN, Gopurenko D, Bulut Z, Dewoody JA (2009) Condition-dependent mate choice and a reproductive disadvantage for MHC-divergent male tiger salamanders. Mol Ecol 18:33073315

Bush SJ, Castillo-Morales A, Tovar-Corona JM, Chen L, Kover PX, Urrutia AO (2014) Presence-absence variation in A. thaliana is primarily associated with genomic signatures consistent with relaxed selective constraints. Mol Biol Evol 31:59-69

Chain FJJ, Evans BJ (2006) Multiple mechanisms promote the retained expression of gene duplicates in the tetraploid frog Xenopus laevis. Plos Genet 2:478-490

Chain FJ, Dushoff J, Evans BJ (2011) The odds of duplicate gene persistence after polyploidization. BMC Gen 12:599

Chen YY, Zhang YY, Zhang HM, Ge YF, Wan QH, Fang SG (2010) Natural selection coupled with intragenic recombination shapes diversity patterns in the Major Histocompatibility Complex class II genes of the giant panda. J Exp Zool B Mol Dev Evol 314:208-223

Chida AS, Goyos A, Robert J (2011) Phylogenetic and developmental study of CD4, CD8 alpha and beta T cell co-receptor homologs in two amphibian species, Xenopus tropicalis and Xenopus laevis. Dev Comp Immunol 35:366-377

Comai L, Tyagi AP, Winter K, Holmes-Davis R, Reynolds SH, Stevens Y, Byers B (2000) Phenotypic instability and rapid gene silencing in newly formed Arabidopsis allotetraploids. Plant Cell 12:1551-1567

Delport W, Poon AFY, Frost SDW, Pond SLK (2010) Datamonkey 2010: a suite of phylogenetic analysis tools for evolutionary biology. Bioinformatics 26:2455-2457

Du Pasquier L, Blomberg B (1982) The expression of antibody diversity in natural and laboratory-made polyploid individuals of the clawed toad Xenopus. Immunogenetics 15:251-260

Eimes JA, Bollmer JL, Whittingham LA, Johnson JA, Van Oosterhout C, Dunn PO (2011) Rapid loss of MHC class II variation in a bottlenecked population is explained by drift and loss of copy number variation. J Evol Biol 24:1847-1856

Eizaguirre C, Yeates SE, Lenz TL, Kalbe M, Milinski M (2009) MHCbased mate choice combines good genes and maintenance of MHC polymorphism. Mol Ecol 18:3316-3329

Evans BJ (2007) Ancestry influences the fate of duplicated genes millions of years after polyploidization of clawed frogs (Xenopus). Genetics 176:1119-1130

Evans BJ, Morales JC, Picker MD, Kelley DB, Melnick DJ (1997) Comparative molecular phylogeography of two Xenopus species, $X$. gilli and X. laevis, in the south-western Cape province, South Africa. Mol Ecol 6:333-343

Evans BJ, Kelley DB, Tinsley RC, Melnick DJ, Cannatella DC (2004) A mitochondrial DNA phylogeny of African clawed frogs: phylogeography and implications for polyploid evolution. Mol Phylogen Evol 33:197-213 
Excoffier L, Lischer HE (2010) Arlequin suite ver 3.5: a new series of programs to perform population genetics analyses under Linux and Windows. Mol Ecol Resour 10:564-567

Fay JC, Wu CI (1999) A human population bottleneck can account for the discordance between patterns of mitochondrial versus nuclear DNA variation. Mol Biol Evol 16:1003-1005

Figueroa F, Mayer WE, Sato A, Zaleska-Rutczynska Z, Hess B, Tichy H, Klein J (2001) Mhc class I genes of swordtail fish, Xiphophorus: variation in the number of loci and existence of ancient gene families. Immunogenetics 53:695-708

Flajnik MF (1996) The immune system of ectothermic vertebrates. Vet Immunol Immunopath 54:145-150

Flajnik MF, Kasahara M (2001) Comparative genomics of the MHC: glimpses into the evolution of the adaptive immune system. Immunity 15:351-362

Flajnik MF, Kasahara M (2010) Origin and evolution of the adaptive immune system: genetic events and selective pressures. Nat Rev Genet 11:47-59

Forsberg LA, Dannewitz J, Petersson E, Grahn M (2007) Influence of genetic dissimilarity in the reproductive success and mate choice of brown trout-females fishing for optimal MHC dissimilarity. J Evol Biol 20:1859-1869

Furman BLS, Bewick AJ, Harrison TL, Greenbaum E, Gvoždík V, Kusamba C, Evans B (2015) Pan-African phylogeography of a model organism, the African clawed frog Xenopus laevis. Mol Ecol 24(4):909-925

Garrigan D, Hedrick PN (2001) Class I MHC polymorphism and evolution in endangered California Chinook and other Pacific salmon. Immunogenetics 53:483-489

Gerhardt HC (1991) Female mate choice in treefrogs: static and dynamic acoustic criteria. Anim Behav 42:615-635

Gurdon JB (1996) Introductory comments: Xenopus as a laboratory animal. In: Tinsley RC, Kobel HR (eds) The biology of Xenopus. Oxford University Press, London, pp 3-6

Hauswaldt JS, Stuckas H, Pfautsch S, Tiedemann R (2007) Molecular characterization of MHC class II in a nonmodel anuran species, the fire-bellied toad Bombina bombina. Immunogenetics 59:479-491

Hellsten U, Khokha MK, Grammer TC, Harland RM, Richardson P, Rokhsar DS (2007) Accelerated gene evolution and subfunctionalization in the pseudotetraploid frog Xenopus laevis. BMC Biol 5:31

Hudson RR, Kreitman M, Aguade M (1987) A test of neutral molecular evolution based on nucleotide data. Genetics 116:153-159

Hughes AL, Hughes MK, Watkins DI (1993) Contrasting roles of interallelic recombination at the HLA-A and HLA-B loci. Genetics 133:669-680

Jackson JA, Tinsley RC (2001) Host-specificity and distribution of cephalochlamydid cestodes: correlation with allopolyploid evolution of pipid anuran hosts. J Zool 254:405-419

Jackson JA, Tinsley RC (2003) Parasite infectivity to hybridising host species: a link between hybrid resistance and allopolyploid speciation? Int J Parasitol 33:137-144

Kamiya T, O’Dwyer K, Westerdahl H, Senior A, Nakagawa S (2014) A quantitative review of MHC-based mating preference: the role of diversity and dissimilarity. Mol Ecol 23:5151-5163

King KC, Seppälä O, Neiman M (2012) Is more better? Polyploidy and parasite resistance. Biol Let 8:598-600

Klein SL et al (2002) Genetic and genomic tools for Xenopus research: The NIH Xenopus initiative. Develop Dyn 225:384-391

Klump GM, Gerhardt HC (1987) Use of non-arbitrary acoustic criteria in mate choice by female gray treefrogs. Nature 326:286-288

Kobari F et al (1995) Exon-intron organization of Xenopus MHC class II beta chain genes. Immunogenetics 42:376-385

Kobel HR, Du Pasquier L (1986) Genetics of polyploid Xenopus. Trends Genet 2:310-315
Kruiswijk CP, Hermsen T, Fujiki K, Dixon B, Savelkoul HFJ, Stet RJM (2004) Analysis of genomic and expressed major histocompatibility class Ia and class II genes in a hexaploid Lake Tana African "large" barb individual. Immunogenetics 55:770-781

Librado P, Rozas J (2009) DnaSP v5: a software for comprehensive analysis of DNA polymorphism data. Bioinformatics 25:1451-1452

Lillo F, Faraone FP, Lo Valvo M (2011) Can the introduction of Xenopus laevis affect native amphibian populations? Reduction of reproductive occurrence in presence of the invasive species. Biol Invasions 13:1533-1541

Lillo F, Dufresnes C, Faraone FP, Lo Valvo M, Stock M (2013) Identification and potential origin of invasive clawed frogs Xenopus (Anura: Pipidae) in Sicily based on mitochondrial and nuclear DNA. Ital J Zool 80:566-573

Llaurens V, McMullan M, van Oosterhout C (2012) Cryptic MHC polymorphism revealed but not explained by selection on the class IIB peptide-binding region. Mol Biol Evol 29:1631-1644

Maniero GD, Morales H, Gantress J, Robert J (2006) Generation of a long-lasting, protective, and neutralizing antibody response to the ranavirus FV3 by the frog Xenopus. Dev Comp Immunol 30:649657

Marsden CD et al (2009) Highly endangered African wild dogs (Lycaon pictus) lack variation at the major histocompatibility complex. J Hered 100:S54-S65

Martinsohn JT, Sousa AB, Guethlein LA, Howard JC (1999) The gene conversion hypothesis of MHC evolution: a review. Immunogenetics 50:168-200

McDonald JH, Kreitman M (1991) Adaptive protein evolution at the Adh locus in Drosophila. Nature 351:652-654

Measey GJ et al (2012) Ongoing invasions of the African clawed frog, Xenopus laevis: a global review. Biol Invasions 14:2255-2270

Milinski M (2003) The function of mate choice in sticklebacks: optimizing MHC genetics. J Fish Biol 63(Supplement A):1-16

Miller HC, Moore JA, Nelson NJ, Daugherty CH (2009) Influence of major histocompatibility complex genotype on mating success in a free-ranging reptile population. Proc Roy Soc B 276:1695-1704

Moriarty EC, Cannatella DC (2004) Phylogenetic relationships of the North American chorus frogs (Pseudacris: Hylidae). Mol Phylogen Evol 30:409-420

Morin RD et al (2006) Sequencing and analysis of 10,967 full-length cDNA clones from Xenopus laevis and Xenopus tropicalis reveals post-tetraploidization transcriptome remodeling. Genome Res 16: 796-803

Murray BW, Shintani S, Sültmann H, Klein J (2000) Major Histocompatibility Complex class II A genes in cichlid fishes: identification, expression, linkage relationships, and haplotype variation. Immunogenetics 51:576-586

Neff BD, Garner SR, Heath JW, Heath D (2008) The MHC and nonrandom mating in a captive population of Chinook salmon. Heredity 101:175-185

Nei M (1987) Molecular evolutionary genetics. Columbia University Press, New York

Nuismer SL, Thompson JN (2001) Plant polyploidy and non-uniform effects on insect herbivores. Proc R Soc Lond B 268:1937-1940

Ohta T (1991) Role of diversifying selection and gene conversion in evolution of Major Histocompatibility Complex loci. Proc Natl Acad Sci U S A 88:6716-6720

Ohta Y, Goetz W, Hossain MZ, Nonaka M, Flajnik MF (2006) Ancestral organization of the MHC revealed in the amphibian Xenopus. J Immunol 176:3674-3685

Pandit MK, Pocock MJO, Kunin WE (2011) Ploidy influences rarity and invasiveness in plants. J Ecol 99:1108-1115

Piertney SB, Oliver MK (2006) The evolutionary ecology of the Major Histocompatibility Complex. Heredity 96:7-21 
Pond SLK, Frost SDW (2005) Not so different after all: a comparison of methods for detecting amino acid sites under selection. Mol Biol Evol 22:1208-1222

Pond KSL, Frost SDW, Muse SV (2005) HyPhy: hypothesis testing using phylogenies. Bioinformatics 21:676-679

Pond SLK, Posada D, Gravenor MB, Woelk CH, Frost SDW (2006) GARD: a genetic algorithm for recombination detection. Bioinformatics 22:3096-3098

Pond SLK, Murrell B, Fourment M, Frost SDW, Delport W, Scheffler K (2011) A random effects branch-site model for detecting episodic diversifying selection. Mol Biol Evol 28:3033-3043

Potts WK, Manning CJ, Wakeland EK (1994) The role of infectious disease, inbreeding and mating preferences in maintaining MHC genetic diversity: an experimental test. Philos Trans R Soc Lond B Biol Sci 346:369-378

Reusch TBH, Haberli MA, Aeschlimann PB, Milinski M (2001) Female sticklebacks count alleles in a strategy of sexual selection explaining MHC polymorphism. Nature 414:300-302

Richardson DS, Komdeur J, Burke T, von Schantz T (2005) MHC-based patterns of social and extra-pair mate choice in the Seychelles warbler. Proc R Soc Lond B Biol Sci 272:759-767

Robert J, Cohen N (2011) The genus Xenopus as a multispecies model for evolutionary and comparative immunobiology of the 21 st century. Dev Comp Immunol 35:916-923

Robert J, Ohta Y (2009) Comparative and developmental study of the immune system in Xenopus. Develop Dyn 238:1249-1270

Robert J, Abramowitz L, Gantress J, Morales HD (2007) Xenopus laevis: a possible vector of ranavirus infection? J Wildlife Dis 43:645-652

Roberts SC, Little AC, Gosling LM, Jones BC, Perrett DI, Carter V, Petrie M (2005) MHC-assortative facial preferences in humans. Biol Let 1: 400-403

Sammut B, Marcuz A, Pasquier L (2002) The fate of duplicated major histocompatibility complex class Ia genes in a dodecaploid amphibian, Xenopus ruwenzoriensis. Eur J Immunol 32:2698-2709

Sato K, Flajnik MF, Du Pasquier L, Katagiri M, Kasahara M (1993) Evolution of the MHC: isolation of class II beta-chain cDNA clones from the amphibian Xenopus laevis. J Immunol 150:2831-2843

Sato A, Klein D, Sultmann H, Figueroa F, O’hUigin C, Klein J (1997) Class I Mhc genes of cichlid fishes: identification, expression, and polymorphism. Immunogenetics 46:63-72

Sato A et al (2002) Persistence of MHC heterozygosity in homozygous clonal killifish, Rivulus marmoratus: implications for the origin of hermaphroditism. Genetics 162:1791-1803

Schoen DJ, Burdon JJ, Brown AH (1991) Resistance of Glycine tomentella to soybean leaf rust Phakopsora pachyrhizi in relation to ploidy level and geographic distribution. Theor Appl Genet 83: 827-832

Schwensow N, Eberle M, Sommer S (2008) Compatibility counts: MHCassociated mate choice in a wild promiscuous primate. Proc R Soc Lond B Biol Sci 275:555-564

Semon M, Wolfe KH (2008) Preferential subfunctionalization of slowevolving genes after allopolyploidization in Xenopus laevis. Proc Natl Acad Sci U S A 105:8333-8338
Setchell JM, Charpentier MJ, Abbott KM, Wickings EJ, Knapp LA (2010) Opposites attract: MHC-associated mate choice in a polygynous primate. J Evol Biol 23:136-148

Shen J, Araki H, Chen L, Chen JQ, Tian D (2006) Unique evolutionary mechanism in R-genes under the presence/absence polymorphism in Arabidopsis thaliana. Genetics 172:1243-1250

Shum BP, Avila D, Du Pasquier L, Kasahara M, Flajnik MF (1993) Isolation of a classical MHC class I cDNA from an amphibian. Evidence for only one class I locus in the Xenopus MHC. J Immunol 151:5376-5386

Spurgin LG, van Oosterhout C, Illera JC, Bridgett S, Gharbi K, Emerson BC, Richardson DS (2011) Gene conversion rapidly generates Major Histocompatibility Complex diversity in recently founded bird populations. Mol Ecol 20:5213-5225

Tajima F (1989) Statistical method for testing the neutral mutation hypothesis by DNA polymorphism. Genetics 123:585-595

Tamura K, Stecher G, Peterson D, Filipski A, Kumar S (2013) MEGA6: Molecular Evolutionary Genetics Analysis version 6.0. Mol Biol Evol 30:2725-2729

Thompson JN, Nuismer SL, Merg K (2004) Plant polyploidy and the evolutionary ecology of plant/animal interactions. Biol J Linn Soc 82:511-519

Tinsley RC, Kobel HR (1996) The biology of Xenopus. In: Symposia of the Zoological Society of London, vol 68. Oxford University Press and The Zoological Society of London, Oxford

Tinsley R et al (2012) Acquired immunity protects against helminth infection in a natural host population: long-term field and laboratory evidence. Int J Parasitol 42:931-938

Tinsley RC, Coxhead PG, Stott LC, Tinsley MC, Piccinni MZ, Guille MJ (2015a) Chytrid fungus infections in laboratory and introduced populations: assessing the risks for U.K. native amphibians. Biol Conserv 184:380-388

Tinsley RC, Stott LC, Viney ME, Mable BK, Tinsley MC (2015b) Extinction of an introduced warm climate alien species, Xenopus laevis, by extreme weather events. Biol Invasions. doi:10.1007/ s10530-015-0944-x

van Oosterhout C (2009) A new theory of MHC evolution: beyond selection on the immune genes. Proc R Soc Lond B Biol Sci 276:657-665

van Oosterhout C, Joyce DA, Cummings SM (2006) Evolution of MHC class IIB in the genome of wild and ornamental guppies, Poecilia reticulata. Heredity 97:111-118

Watterson G (1975) On the number of segregating sites in genetical models without recombination. Theoretical Population Genetics 7: 256-276

Wedekind C, Walker M, Portmann J, Cenni B, Muller R, Binz T (2004) MHC-linked susceptibility to a bacterial infection, but no MHClinked cryptic female choice in whitefish. J Evol Biol 17:11-18

Weldon C, du Preez LH, Hyatt AD, Muller R, Speare R (2004) Origin of the amphibian chytrid fungus. Emerg Infect Dis 10:2100-2105

Zelano B, Edwards SV (2002) An MHC component to kin recognition and mate choice in birds: predictions, progress, and prospects. Am Nat 160:S225-S237

Zou J, Secombes CJ (2011) Teleost fish interferons and their role in immunity. Dev Comp Immunol 35:1376-1387 